\title{
Phenotypic Variation for Downy Mildew Resistance among Inbred Broccoli
}

\author{
Min Wang, Mark W. Farnham, and Claude E. Thomas ${ }^{1}$ \\ U.S. Department of Agriculture, Agricultural Research Service, U.S. Vegetable \\ Laboratory, 2875 Savannah Highway, Charleston, SC 29414
}

Additional index words. Brassica oleracea, Peronospora parasitica, doubled-haploids, reaction phenotype $(\mathrm{RP})$

\begin{abstract}
Downy mildew, caused by Peronospora parasitica (Pers. Fr.) Fr., is one of the most economically important diseases of broccoli (Brassica oleracea L. Italica group). Previous studies have shown that resistance to downy mildew in broccoli is dependent on plant age with seedling resistance being independent of mature-plant resistance. The objectives of this study were to: 1) determine if valid evaluations for downy mildew resistance can be conducted at both the cotyledon and the three to four true-leaf stages on the same plants of a given broccoli entry; 2) determine if doubled-haploid (DH) lines derived from the resistant hybrid 'Everest' also exhibit resistance to downy mildew and if so, characterize the resistance phenotype(s) in these lines; and 3) determine if identified resistant DH lines exhibit resistance to isolates of $P$. parasitica acquired from different geographic regions of the United States. Twenty-three DH broccoli inbreds and two commercial hybrids were evaluated for reaction at different developmental stages to infection by $\boldsymbol{P}$. parasitica in a controlled environment. Results showed that broccoli plants can be evaluated for downy mildew resistance in a two-stage process. Inoculation at the cotyledon stage did not offer any cross-protection or otherwise influence the expression of reaction phenotype (RP) when the same plants were subsequently inoculated at the three to four true-leaf stage. Three different RPs to infection by $P$. parasitica were identified in DH inbreds. These were: 1) susceptibility at both the cotyledon stage and the true-leaf stage; 2 ) resistance at both the cotyledon and true-leaf stage; and 3) susceptibility at the cotyledon stage but resistance at the true-leaf stage. There was no effect of two pathogenic isolates from different geographic regions on RP of DH broccoli inbreds. Selection of plant resistance to downy mildew at the cotyledon stage will effectively identify plants with high levels of resistance at subsequent developmental stages.
\end{abstract}

Downy mildew, caused by the obligate parasite, $P$. parasitica, is one of the most important diseases of broccoli and other $B$. oleracea vegetable crops. This disease is distributed worldwide on B. oleracea and is commonly found wherever these crops are grown (Channon, 1981). Infection by $P$. parasitica is favored by day temperatures between 20 and $24{ }^{\circ} \mathrm{C}$ in conjunction with night temperatures between 8 and $16^{\circ} \mathrm{C}$ and conditions of high humidity [relative humidity $(\mathrm{RH})>80 \%$ ], such as frequent rain showers or heavy dews that result in prolonged periods of leaf wetness (Channon, 1981; Kucharek, 1985). Broccoli is susceptible to infection by $P$. parasitica at any stage of

Received for publication 23 Aug. 1999. Accepted for publication $29 \mathrm{Dec}$. 1999. The contents of this publication do not necessarily reflect the views or policies of the U.S. Dept. of Agriculture, nor does mention of trade names, commercial products, or organizations imply endorsement by the U.S. Government. The cost of publishing this paper was defrayed in part by the payment of page charges. Under postal regulations, this paper therefore must be hereby marked advertisement solely to indicate this fact.

${ }^{1}$ Research Geneticist, Research Geneticist, and Research Plant Pathologist, respectively. growth. Infection at the seedling stage is particularly devastating because cotyledons and young leaf tissues are highly susceptible and seedlings are stunted or killed. Infections at this stage reduce stand and, ultimately, yield. Broccoli becomes less susceptible after the seedling stage (Davison, 1966; Dickinson and Greenhalgh, 1977; Dickson and Petzoldt, 1993), but infection of mature plants can occur with visible or latent symptoms on heads, ruining their market quality or storability (Channon, 1981; Sherf and MacNab, 1986).

Peronospora parasitica is an intercellular parasite with large globular to coarse-lobed haustoria. The pathogen produces soilborne oospores and airborne conidia, the infective spore form (Williams, 1986). It has a relatively wide host range, infecting not only $B$. oleracea, but almost all cultivated and some weed species in the family Brassicaceae (Dickinson and Greenhalgh, 1977; McMeekin, 1969; Sherf and MacNab, 1986). De Bruyn (1937) and McMeekin (1960) suggested that heterothallism may give this pathogen substantial capacity for genetic variation. Differential interaction between $B$. olerace $a$ hosts and $P$. parasitica isolates has been reported by several groups (Davison, 1966; Dias et al., 1993; Dickinson and Greenhalgh, 1977; Greenhalgh and
Dickinson, 1975, 1976; Leckie et al., 1998). Three different races of $P$. parasitica, designated race 1 , race 2 , and race 3 , have been cited for B. oleracea (Natti et al., 1967; Dickson and Petzoldt, 1993). At present, however, characterization of current races of $P$. parasitica and their relationships to previously identified races are nearly impossible because established differential lines for screening isolates are not available.

Fungicides provide significant control of downy mildew (Brophy and Laing, 1992). In a survey of California broccoli producers in 1991, about two-thirds of respondents indicated that they sprayed broccoli regularly with fungicides to control this disease (Flint et al., 1992). Recently, seed treatment with "CGA245704," an activator of systemic acquired resistance, reduced sporulation of $P$. parasitica in B. oleracea; however, further testing is needed to fully determine the usefulness of this compound(Jensen, et al., 1998). Given concerns over environmental safety, the potential loss of registered fungicides (Flint et al., 1992), and the reported (Crute et al., 1985, 1994) development of P. parasitica resistance to phenylamide fungicides, the development and cultivation of resistant broccoli cultivars are attractive alternative methods to control downy mildew on this crop.

Natti et al. (1967) found resistance at the cotyledon-stage to downy mildew, race 1 , in PI 189028 and determined that a single dominant gene controlled this resistance. They also found that PI 208478 had resistance at the cotyledon stage to race 2 that was controlled by a different single dominant gene. Conversely, HoserKrause et al.(1987) described a single recessive gene responsible for downy mildew resistance against a Polish isolate of $P$. parasitica at the four to five true-leaf stage in a broccoli line originating in the United States. Other studies have evaluated downy mildew resistance in broccoli among U.S. Plant Introductions (Thomas and Jourdain, 1990), commercial cultivars and experimental lines (Laemmlen and Mayberry, 1984), and accessions from world collections (Coelho et al., 1998). However, none of these latter reports characterized the inheritance of the resistance described.

Dickson and Petzoldt (1993) showed that the $F_{1}$ hybrid cultivar Everest has a relatively high level of resistance to race 2 at the true-leaf stage. Personal communications from several commercial broccoli breeders indicate that 'Everest' is utilized as a source of downy mildew resistance in the development of new cultivars. We know of no published reports on inheritance of resistance to downy mildew in 'Everest'. In addition, commercial protection of the parents of 'Everest' as proprietary materials prevents the use of the putative resistant parent(s) of this $F_{1}$ hybrid to determine the mode of inheritance. Thus, we have developed doubled-haploid(DH) lines from 'Everest' with the goal of producing homozygous resistant lines (Farnham, 1998; Wang and Farnham, 1999). In the present study, one objective was to determine if valid evaluations for downy mildew resistance could be conducted at both the cotyledon stage and the true-leaf stage on the same plants of a given broccoli entry. A second 
objective was to determine if DH lines derived from 'Everest' exhibit resistance to downy mildew, and, if so, to characterize the phenotypic expression of resistance in these lines. A final objective was to determine if resistant DH lines exhibit resistance to isolates of $P$. parasitica from different geographic regions of the United States.

\section{Materials and Methods}

Plant material. Twenty-three inbreds were utilized in a series of experiments. All of these inbreds were DH lines developed using standard techniques of anther culture at the U.S. VegetableLaboratory (USVL), Charleston, S.C. (Farnham, 1998). The lines were given USVL designations as follows: USVL007, 012, 013, 014, 016, 079, 080, 081, 082, 083, 084, 085, 086, 087, 088, 089, 090, 091, and 092, all derived from the hybrid cultivar Everest(Rogers Seed Co., Gilroy, Calif.); USVL039, derived from the hybrid cultivar High Sierra (Asgrow Seed Co., San Juan Bautista, Calif.); and USVL047, 077, and 078, derived from the hybrid cultivar Marathon (Sakata Seed, Salinas, Calif.). Lines derived from 'Everest' were expected to exhibit varying levels of resistance to downy mildew, while lines derived from 'High Sierra' or 'Marathon' were not expected to exhibit resistance. 'Everest' was included in all tests as a resistant check, and the hybrid 'Green Valiant' was included as a susceptible check in the first experiment. Seeds of all inbreds used in experiments were produced at the USVL, while hybrid seed were obtained from the companies that developed them.

Growing conditions. All experiments were conducted from Nov. 1997 to Dec. 1998 in a walk-in growth room at a temperature of $22^{\circ} \mathrm{C}$ and a 14 -h photoperiod supplying $\approx 240$ $\mu \mathrm{E} \cdot \mathrm{m}^{-2} \cdot \mathrm{s}^{-1}$ from sodium vapor lamps. In each experiment, seeds of each entry were sown in 10-cm plastic pots filled with Metro Mix 360 (Grace Sierra, Milpitas, Calif.). For experimental purposes, two stages of plant development were identified for inoculation with $P$. parasitica. These stages were 1 ) cotyledon stage $=$ cotyledons fully expanded, no true leaves present; and 2) true-leaf stage = plants with three to four expanded leaves.

Pathogen source and inoculum preparation. Two isolates of $P$. parasitica, designated $\mathrm{SC} 1$ and $\mathrm{CA} 1$, were used. Isolate SC1 was previously utilized and described as a race 2 isolate of $P$. parasitica by Thomas and Jourdain (1990), who originally isolated it from fieldgrown plants of broccoli cv. Calabrese at Charleston, S.C., in 1987. Isolate CA1 was obtained from Joseph Stern (Orsetti Seed Co., Hollister, Calif.) who isolated it from infected broccoli leaf tissues from a field in San Juan Bautista, Calif. The race specificity of CA1 is unknown. SC1 and CA1 were each maintained independently on cotyledons of the susceptible broccoli cv. Green Duke with reinoculations every $7 \mathrm{~d}$.

For preparation of inoculum, cotyledons of 'Green Duke' with abundant sporulation of $P$. parasitica were collected and placed in $50 \mathrm{~mL}$ deionized water in a $250-\mathrm{mL}$ flask. The flask was covered and shaken vigorously $50-80$ times to dislodge the conidia. The resultant spore suspensions were filtered through two layers of cheesecloth to remove plant debris. Spore concentrations of all inoculum suspensions were determined using a hemacytometer and adjusted to $1.0 \times 10^{4}$ spores/mL by dilution with deionized water.

Inoculation and evaluation. The entire adaxial and abaxial surfaces of all cotyledons inoculated at the cotyledon stage or leaves inoculated at the true-leaf stage were sprayed uniformly with inoculum using a Paasche Type$\mathrm{H}$ airbrush (Paasche Airbrush Co., Chicago) at $138 \mathrm{kPa}$. Immediately after inoculation, plants were placed in a dark dew chamber at $16^{\circ} \mathrm{C}$ and $100 \% \mathrm{RH}$ for $24 \mathrm{~h}$ to stimulate germination of spores. Plants were then returned to the growth room at conditions described previously. After $6 \mathrm{~d}$ in the growth room, inoculated plants were again placed in the dew chamber for $24 \mathrm{~h}$ to promote sporulation of $P$. parasitica on the inoculated leaves. After plants were removed from the dew chamber the second time, they were evaluated for downy mildew reaction phenotype.

Both cotyledons, following inoculation at the cotyledon stage, and the first two trueleaves, following inoculation at the true-leaf stage, were observed on each plant under a stereo microscope at magnification up to $\times 65$. Ratings of RP were assigned using the following $0-9$ scale: $0=$ no symptoms; $1=$ small necrotic flecks, no sporulation; $3=$ necrotic flecks, one to few sporangiophores present; $5=$ necrotic lesions, sparse, scattered sporulation usually confined to necrotic areas; $7=$ necrotic lesions, sometimes with accompanying chlorosis, scattered, heavy to abundant sporulation in both chlorotic and necrotic areas; $9=$ necrosis and some chlorosis may be evident, uniformly heavy sporulation over abaxial, adaxial, or both surfaces.

Plants were grouped into three classes for reaction to infection by $P$. parasitica based on their RP ratings. These classes were: 1) highly resistant $=\mathrm{RP}<3$;2) moderately resistant $=\mathrm{RP}$ = 3-5; and 3) susceptible $=\mathrm{RP}>5$.

Expt. 1. Seven entries (USVL012, 039, 047, 081, 086; 'Everest' and 'Green Valiant') were used to determine: 1) if evaluation could be conducted on the same plants at both the cotyledon stage and the true-leaf stage; and 2) whether inoculation at the cotyledon stage would affect subsequent inoculation at the true-leaf stage. All experimental plants were seeded at the same time. There were three inoculation stage treatments. One-third of the plants of each entry were inoculated at the cotyledon stage only and subsequently evaluated. Another third were inoculated and subsequently evaluated at the true-leaf stage only. A final third were inoculated at both the cotyledon and the true-leaf stages and subsequently evaluated at the true-leaf stage. Overall, there were 21 different treatments. All treatments were arranged in a randomized complete-block (RCB) design with three blocks. Three seedlings were used for each treatment per block. Only isolate $\mathrm{SC} 1$ of $P$. parasitica was used as the source of inoculum.
As an extension of Expt. 1, $\approx 20$ 'Everest'derived lines were evaluated for their RP to $P$. parasitica infection following inoculation at the cotyledon and true-leaf stages. Lines were identified that expressed variable levels of resistance at a particular stage and variable levels of resistance between stages. These were included in the subsequent replicated tests.

Expt. 2. The purposes of this experiment were to further evaluate lines with different resistant phenotypes to downy mildew and to confirm that inoculation with $P$. parasitica at the cotyledon stage had no effect on symptom expression from subsequent inoculation at the true-leaf stage. Ten entries (USVL012, 047, 077, 078, 088, 089, 090, 091, 092, and 'Everest') were included, with three different inoculation treatments as described for Expt. 1. Overall, there were 30 different treatments. All treatments were arranged in a RCB design with three blocks. Two seedlings were used for each treatment per block. Isolate SC1 was used as the inoculum for $P$. parasitica. USVL012 and 'Everest' were included in this experiment as resistant checks and USVL047 was included as a susceptible check.

Expt. 3. Eight entries (USVL012, 047, 077, 078, 090, 091, 092, and 'Everest') were used to determine if 'Everest'-derived DH lines exhibiting different resistance phenotypes had consistent reactions to isolates of $P$. parasitica from different geographic regions. One set of the eight entries was inoculated with isolate $\mathrm{SC} 1$ at the cotyledon stage and another set at the true-leaf stage. This same inoculation procedure was also performed with isolate CA1 on separate sets of the eight entries. Overall, this gave a total of 32 different treatments. All treatments were arranged in an RCB design with three blocks. Three seedlings were used for each treatment per block.

Statistical analysis. All data on RP were collected on an individual plant basis in all experiments, and each experiment was analyzed using analysis of variance (ANOVA) (SAS Institute, 1986). Treatment means were compared using a Fisher's protected least significant difference test (LSD) $(P \leq 0.05)$.

\section{Results}

Expt. 1. Plants were more resistant at the true-leaf stage than at the cotyledon stage (Fig. 1). The mean for an inoculation at the true-leaf stage $(\mathrm{RP}$ rating $=4.8)$ was significantly $(P \leq 0.0001)$ lower than that for the inoculation at the cotyledon stage (RP rating $=7.8$ ). When evaluated at the true-leaf stage, there was no difference between plants that were inoculated at both the cotyledon stage and the true-leaf stage $(\mathrm{RP}=4.8)$ and those that were inoculated only at the true-leaf stage $(\mathrm{RP}=4.8)$. However, RP means among entries for a given inoculation treatment were significantly $(P \leq 0.0001)$ different. At the cotyledon stage, RP means ranged from 4.4 (USVL012) to 9.0 (USVL039). No highly resistant entry was identified at this stage. At the true-leaf stage, USVL012 was the most resistant entry, and USVL039 the most 
susceptible entry with RP means of 1.7 and 7.8 , respectively. A significant $(P \leq 0.0001)$ interaction between inoculation treatment and entry was indicated by ANOVA.

Expt. 2. As in Expt. 1, plants were more resistant at the true-leaf stage than at the cotyledon stage (Fig. 2). Inoculation at the true-leaf stage resulted in an overall mean RP (3.1) that was significantly $(P \leq 0.0001)$ lower than the overall mean for inoculation at the cotyledon stage $(\mathrm{RP}=5.0)$. Also, as observed in Expt. 1, when evaluated at the true-leaf stage, the difference between plants that were inoculated at both the cotyledon stage and the true-leaf stage $(\mathrm{RP}=3.0)$, and those that were inoculated only at the true-leaf stage $(\mathrm{RP}=3.1)$ was nonsignificant. In addition, RP means among entries for a given inoculation treatment differed significantly $(P \leq 0.0001)$. In this experiment, a new resistant phenotype, exemplified by entries USVL088, 090, and 091 was observed, which expressed high levels of resistance at both the cotyledon and the true-leaf stages. USVL088 was the most resistant of the three entries at the cotyledon stage with RP mean of 1.8. At the true-leaf stage, USVL088, 090, and 091, similar to their parent 'Everest', were highly resistant with RP means < 1 . The RP means for USVL012 and USVL047, the resistant (trueleaf stage) and susceptible checks, respectively, were similar to those exhibited in Expt. 1. For this experiment ANOVA again revealed a significant $(P \leq 0.0001)$ interaction between inoculation treatment and entry.

Expt. 3. In this experiment, pathogen isolate did not affect RP means, but inoculation treatment and entry effects were highly significant (Table 1). The overall RP means of 3.7 (SC1) and 3.7 (CA1) for inoculation treatment at the true-leaf stage were significantly lower than those at the cotyledon stage (5.3 and 5.2, respectively). RP means differed significantly among entries for a given isolate or inoculation treatment (Fig. 3). In this experiment, two different resistant phenotypes were confirmed among 'Everest'-derived DH lines (Fig. 3). Regardless of the isolate used, USVL090 and 091 exhibited RP representing high levels of resistance at both the cotyledon and the trueleaf stages. Conversely, with both isolates, USVL012 and 092 exhibited RP representing susceptibility or only moderate resistance at the cotyledon stage, but a high level of resistance against both isolates at the true-leaf stage. Other entries (USVL047, 077, and 078) were susceptible at both stages. As observed in all experiments, there was a significant interaction between inoculation treatment and entry (Table 1). However, interactions (isolate $\times$ inoculation treatment, isolate $\times$ entry, and inoculation treatment $\times$ isolate treatment $\times$ entry) were nonsignificant (Table 1).

\section{Discussion}

Studies of host response in $B$. oleracea to inoculation with $P$. parasitica under controlled conditions typically have involved evaluations of plants following inoculation at the cotyledon stage (Dias et al., 1993; Greenhalgh and Dickinson, 1975; Leckie et al., 1998; Natti et

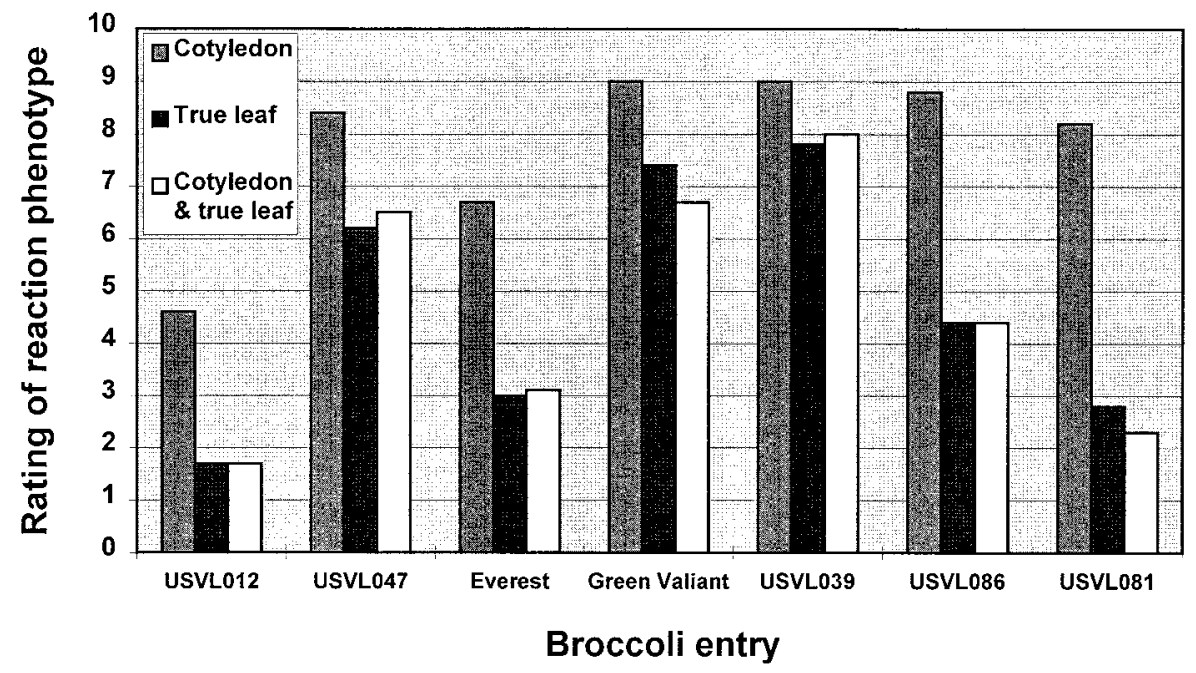

Fig. 1. Mean of reaction phenotype ratings of seven broccoli entries inoculated and evaluated at the cotyledon stage only, at the true leaf stage only, or at both stages using $P$. parasitica isolate $\mathrm{SC} 1$ in Expt. 1. Ratings of reaction phenotype (RP) were scaled from 0 to $9.0=$ No symptoms. $1=$ small necrotic flecks, no sporulation. $3=$ necrotic flecks, one to few sporangiophores present. $5=$ necrotic lesions, sparse scattered sporulation usually confined to necrotic areas. $7=$ necrotic lesions, sometimes with accompanying chlorosis, scattered, heavy to abundant sporulation in both chlorotic and necrotic areas. $9=$ necrosis and some chlorosis may be evident, uniformly heavy sporulation over abaxial, adaxial, or both surfaces.

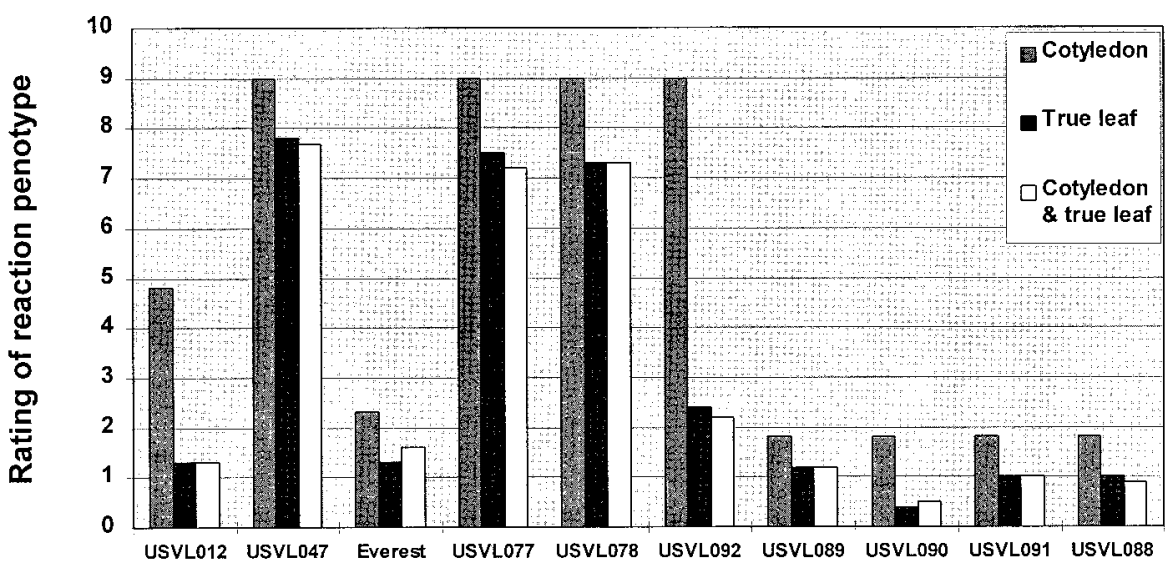

Broocoli entry

Fig. 2. Mean of reaction phenotype ratings of 10 broccoli entries inoculated and evaluated at the cotyledon stage only, at the true leaf stage only, or at both stages using $P$. parasitica isolate $\mathrm{SC} 1$ in Expt. 2. Ratings of reaction phenotype (RP) were scaled from 0 to $9.0=$ No symptoms. $1=$ small necrotic flecks, no sporulation. $3=$ necrotic flecks, one to few sporangiophores present. $5=$ necrotic lesions, sparse scattered sporulation usually confined to necrotic areas. $7=$ necrotic lesions, sometimes with accompanying chlorosis, scattered, heavy to abundant sporulation in both chlorotic and necrotic areas. $9=$ necrosis and some chlorosis may be evident, uniformly heavy sporulation over abaxial, adaxial, or both surfaces.

al., 1967; Silue et al., 1996), at the true-leaf stage (Dickson and Petzoldt, 1993; Hoser-Krauze, et al., 1987; Laemmelen and Mayberry, 1984; Thomas and Jourdain, 1989, 1990), or at the cotyledon and true-leaf stages on different sets of plants of a given entry (Coelho et al., 1998). We are unaware of studies using a two-stage inoculation procedure on the same plants in a controlled environment as described herein. Results of this study show that the same broccoli plants can be evaluated for downy mildew resis- tance in two stages in a controlled environment. In this process, RP can be evaluated following inoculation at the cotyledon stage and again following a second inoculation at the true-leaf stage. In the study reported here, inoculation at the cotyledon stage did not offer any crossprotection or otherwise influence the expression of RP when the same plants were subsequently inoculated at the true-leaf stage. Thus, the two-stage approach described should be particularly useful for evaluation of early gen- 
Table 1. Mean squares (MS) from the analysis of variance for reaction phenotype ratings of eight broccoli entries following inoculation at two stages (cotyledon or true leaf) and with two isolates ( $\mathrm{SC} 1$ and $\mathrm{CA} 1)$ in Expt. 3.

\begin{tabular}{lccc}
\hline \hline Source & df & MS & F value \\
\hline Block & 2 & 0.144 & $1.13^{\mathrm{Ns}}$ \\
Inoculation stage $^{\mathrm{z}}$ & 1 & 58.33 & $454.82^{* * *}$ \\
Isolate $^{\mathrm{y}}$ & 1 & 0.08 & $0.65^{\mathrm{Ns}}$ \\
Entry $_{\text {Inoculation stage } \times \text { isolate }}$ & 7 & 162.79 & $1269.22^{* * *}$ \\
Inoculation stage $\times$ entry & 1 & 0.05 & $0.38^{\mathrm{ss}}$ \\
Isolate $\times$ entry & 7 & 15.60 & $121.58^{* * * *}$ \\
Inoculation stage $\times$ isolate $\times$ entry & 7 & 0.07 & $0.54^{\mathrm{Ns}}$ \\
Error & 7 & 0.02 & $0.19^{\text {ss }}$ \\
Corrected total & 62 & 0.13 & \\
\hline
\end{tabular}

${ }^{\mathrm{z}}$ Inoculation at cotyledon stage or three true-leaf stage.

'Isolate SC1 or CA1.

ss, **** Nonsignificant or significant at $P \leq 0.001$, respectively.

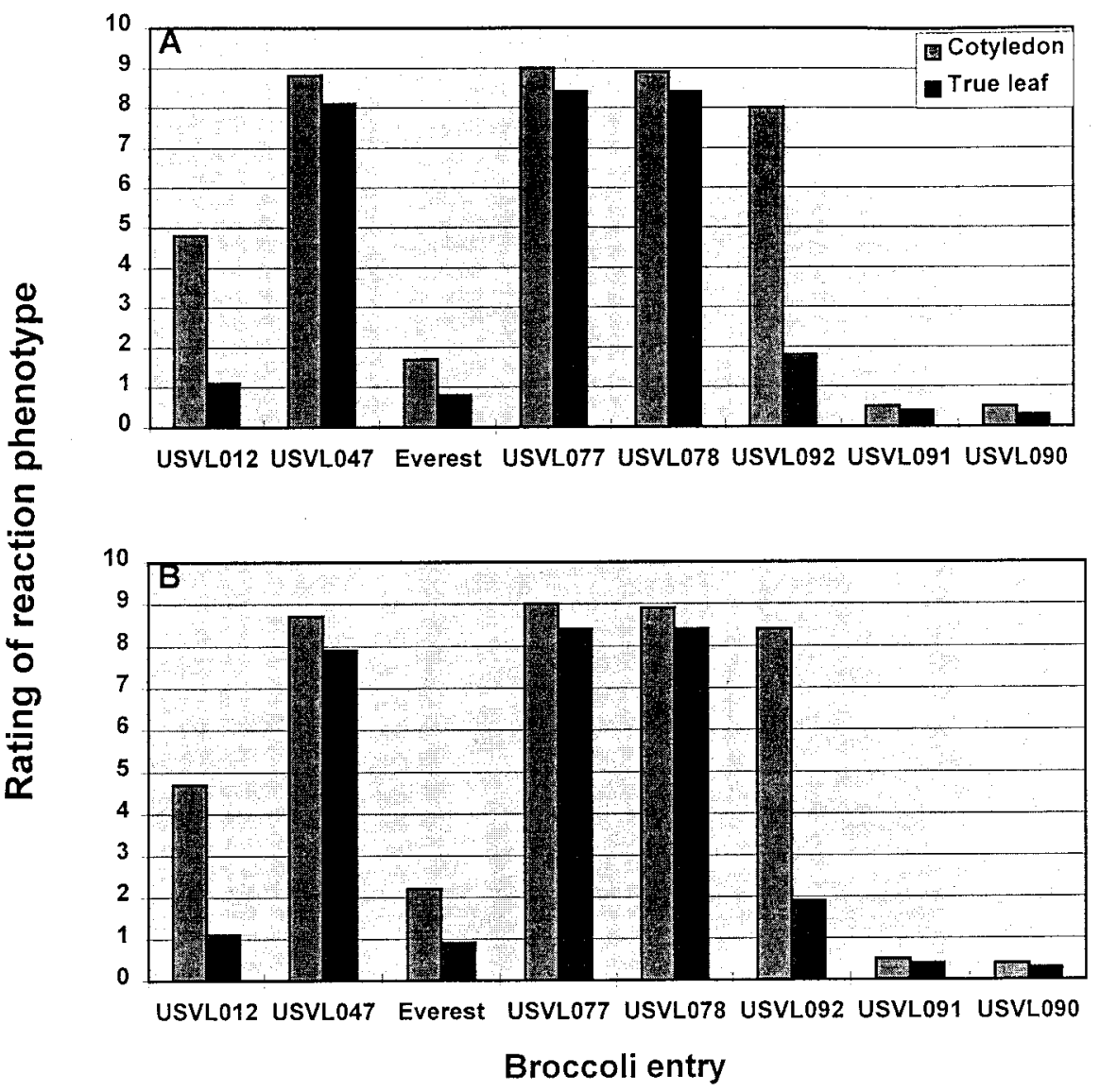

Fig. 3. Mean reaction phenotype ratings of eight broccoli entries inoculated at cotyledon stage or true leaf stage using $P$. parasitica isolates (A) CA1 or (B) SC1 in Expt. 3. Ratings of reaction phenotype (RP) were scaled from 0 to $9.0=$ No symptoms. $1=$ small necrotic flecks, no sporulation. $3=$ necrotic flecks, one to few sporangiophores present. $5=$ necrotic lesions, sparse scattered sporulation usually confined to necrotic areas. $7=$ necrotic lesions, sometimes with accompanying chlorosis, scattered, heavy to abundant sporulation in both chlorotic and necrotic areas. $9=$ necrosis and some chlorosis may be evident, uniformly heavy sporulation over abaxial, adaxial, or both surfaces.

eration selections (e.g., $\mathrm{F}_{2}$ plants) that are typically evaluated in a breeding program and that cannot be easily replicated in time or space. In numerous instances this approach also could save time, money and seed stocks.

All entries evaluated in this study were always less susceptible to downy mildew, i.e., expressed less severe RP, at the true-leaf stage than at the cotyledon stage. Some susceptible (e.g., USVL047) and some resistant entries (e.g., USVL090) exhibited susceptibility or resistance, respectively, at both stages, but RP means were always lower for tests at the trueleaf stage. Other entries (e.g., USVL092) were classified very differently between inoculation stages, because they exhibited highly resistant RP at the true-leaf stage but susceptible $\mathrm{RP}$ at the cotyledon stage. These entries that exhibited RP characteristic of different resistance classes at the two inoculation stages contributed to the significant interaction between inoculation time and entry observed in all experiments (Figs. 1-3). Our results utilizing the same plants tend to agree with those of others who have compared cotyledon and trueleaf stage inoculations on different plants. In general, true leaves have exhibited higher levels of resistance to $P$. parasitica than have cotyledons (Coelho et al., 1998; Davison, 1966; Dickinson and Greenhalgh, 1977; Dickson and Petzoldt, 1993). In this study, three RP were identified: 1) susceptibility at both the cotyledon stage and the true-leaf stage; 2) resistance at both the cotyledon stage and the true-leaf stage; and 3) susceptibility at the cotyledon stage but resistance at the true-leaf stage. We are unaware of any reports and we have not observed an accession or line of $B$. oleracea exhibiting resistance at the cotyledon stage and susceptibility at a true-leaf stage.

The DH-derived inbreds in our study exhibited a wide range of RP means demonstrating significant phenotypic variation to infection by $P$. parasitica. The identification of several uniformly resistant inbreds indicates that 'Everest' is an excellent parental source for developing homozygous lines with downy mildew resistance. Dickson and Petzoldt (1993) reported that 'Everest' was highly susceptible to $P$. parasitica at the cotyledon stage but resistant at the mature plant stage. In Expt. 1, 'Everest' exhibited susceptibility at the cotyledon stage and moderate resistance at the true-leaf stage. However, in Expts. 2 and 3 of this study and other studies not reported herein (unpublished data), higher levels of resistance of 'Everest' at the cotyledon stage and at the true-leaf stage were observed. We surmise that the lack of resistance of 'Everest' in Expt. 1 was atypical. Expt. 1 exhausted the local supply of 'Everest' seed and a new seed supply was obtained for subsequent experiments. This change in seed lot is the only factor that might explain the variable reaction by 'Everest' in these experiments. Importantly, DH lines from the USVL program, including checks (i.e., USVL012 and 047), always had consistent reactions to downy mildew in all experiments. The differences for 'Everest' observed in this study and those reported by Dickson and Petzoldt (1993) could be due to differences between pathogen isolates used in the respective studies. In general, the resistant reaction observed for 'Everest' in Expts. 2 and 3 is similar to that observed by other broccoli breeders in the United States. (personal communication).

Among 'Everest'-derived DH lines evaluated for resistance to downy mildew in this study, two distinctive phenotypes were identified. Lines including USVL090 and 091 were highly resistant at both inoculation stages, while other lines, including USVL012 and 092, were highly resistant only at the true-leaf stage. These distinctive phenotypes were expressed consistently against two geographically divergent isolates of $P$. parasitica. It is noteworthy that the $\mathrm{DH}$ lines with resistance at both stages typically had RP means lower than those of 'Everest' (Figs. 2 and 3). The obser- 
vation of two distinctive resistant phenotypes arising from the hybrid 'Everest' indicates that more than one gene may be contributing to resistance in this hybrid. However, further genetic studies utilizing the DH lines expressing the different resistance phenotypes must be conducted to verify this likelihood. Although not described in these experiments, 'Everest'-derived DH lines that are highly susceptible to downy mildew at all stages are common. This probably indicates that one of the inbred parents of 'Everest' was more susceptible to downy mildew than the other.

In Expt. 3, we used two geographically distinct isolates (SC1 and CA1) of $P$. parasitica. At present, distinguishing between these two isolates as previously cited races is not possible. Although Natti et al. (1967) reported differentiation of Race 1 and Race 2, the differential $B$. oleracea lines these authors used to type isolates were never formally documented or released. Currently, usable and established differential lines for typing $P$. parasitica races are no longer available in the United States. As we have done in this study, private companies that routinely screen for downy mildew resistance in broccoli typically use different isolates from different geographic regions to verify consistent resistance (personal communications). Although we assume that different races of $P$. parasitica exist in the United States, new differential test lines must be identified to confirm this.

Dickson and Petzoldt (1993) tested a set of broccoli entries for downy mildew reaction using isolates sampled from four geographic regions of the United States. They concluded that selecting for high levels of resistance at the true-leaf stage using an isolate from one geographic region should result in selection of good resistance against an isolate from another region. For the phenotypes tested, the results of experiments reported herein using isolates from two different regions support this conclusion. Moreover, and more importantly, results of this study also indicate that selection of broccoli plants exhibiting resistance to downy mildew at the cotyledon stage can be expected to effectively identify plants having high levels of resistance at subsequent stages of development.

\section{Literature Cited}

Brophy, T.F. and M.D. Laing. 1992. Screening of fungicides for the control of downy mildew on container-grown cabbage seedling. Crop Prot. 11:160-164.

Channon, A.G. 1981. Downy mildew of Brassicas, p. 321-339. In: D.M. Spencer (ed.). The downy mildews. Academic, New York.

Coelho, P., D. Leckie, D. Astley, I.R. Crute, K. Bahcevandziev, L. Valerio, A. Monteiro, and I. Boukema. 1998. The relationship between cotyledon and adult plant resistance to downy mildew (Peronospora parasitica) in Brassica oleracea. Acta Hort. 459:335-342.

Crute, I.R., J.M. Norwood, and D.L. Gordon. 1985. Resistance to phenylamide fungicides in lettuce and Brassica downy mildew, p. 311-314. In: Proc. Mixture Centenary Mtg. Bordeaux. Brit. Crop Protection Council Monograph No. 31.

Crute, I.R., P.L. Gordon, and N.A. Moss. 1994. Variation for response to phenylamides in UK populations of Bremia lactucae (lettuce downy mildew) and Peronospora parasitica (Brassica downy mildew), p. 155-162. In: S. Heaney, D.W. Hollomon, M. Smith, P.E. Russell, and D.W. Parry (eds.). Fungicide resistance. Brit. Crop Protection Council Monograph No. 60

Davison, E.M. 1966. Some aspects of the biology of Peronospora parasitica and related organisms. $\mathrm{PhD}$ Diss., Univ. of Bristol, U.K.

De Bruyn, H.L.G. 1937. Heterothallism in Peronospora parasitica. Genetics 19:553-559.

Dias, J.S., M.E. Ferrcira, and P.H. Williams. 1993. Screening of Portuguese cole landraces (Brassica oleracea L.) with Peronospora parasitica and Plasmodiophora brassicae. Euphytica 67:135-141.

Dickinson, C.H. and J.R. Greenhalgh. 1977. Host range and taxonomy of Peronospora on crucifers. Trans. Brit. Mycol. Soc. 69:111-116.

Dickson, M.H., and R. Petzoldt. 1993. Plant age and isolate source affect expression of downy mildew in broccoli. HortScience 28:730-731.

Farnham, M.W. 1998. Doubled-haploid broccoli production using anther culture: Effect of anther source and seed set characteristics of derived lines. J. Amer. Soc. Hort. Sci. 123:73-77.

Flint, M.L., N.A. Davidson, F.G. Zalom, and J.S. Auburn. 1992. Potential losses of pesticides in California and identification of alternative pest management practices in broccoli. Amer. Entomologist Summer:115-121.

Greenhalgh, J.R. and C.H. Dickinson. 1975. Differential reactions of three crucifers to infection by Peronospora parasitica (Pers. ex Fr.) Fr. Phytopathologische Zeitschrift 84:131-141.

Greenhalgh, J.R. and C.H. Dickinson. 1976. Studies of crucifer seedling resistance to Peronospora parasitica. Ann. Appl. Biol. 84:281-287.

Hoser-Krauze, J., E. Lakowska-Ryk, and J. Antosik. 1987. The inheritance of broccoli (Brassica oleracea L. var. botrytis) leaf resistance to downy mildew-Peronospora parasitica (pers.) ex. Fr. Genetica Polonica 28:377-380.

Jensen, B.D., A.O. Latunde-Dada, D. Hudson, and J.A. Lucas. 1998. Protection of Brassica seedlings against downy mildew and damping off by seed treatment with CGA245704, an activator of systemic acquired resistance. Pestic. Sci. 52:63-69.

Kucharek, T. 1985. Downy mildew of crucifers. Plant Pathol. Fact Sheet, Inst. of Food and Agr. Sci., Univ. of Florida, Gainesville.

Laemmlen, F.F. and K.S. Mayberry. 1984. Broccoli resistance to downy mildew. California Agr. (Nov-Dec.): 17.

Leckie, D., N. Cogan, D. Astley, I.R. Crute, I. Boukema, M.Santos, K. Baheevandziev, J.SilvaDias, and A.A. Monteiro. 1998. Differential resistance to Peronospora parasitica and Albugo candida in Brassica oleracea. Acta Hort. 459:357-361.

McMeekin, D. 1960. The role of oospores of Peronospora parasitica in downy mildew of crucifers. Phytopathology 50:93-97.

McMeekin, D. 1969. Other hosts for Peronospora parasitica from cabbage and radish. Phytopathology 59:693-696.

Natti, J.J., M.H. Dickson, and J.D. Atkin. 1967. Resistance of Brassica oleracea varieties to downy mildew. Phytopathology 57:144-147.

SAS Institute. 1986. SAS user's guide: Statistics. 1986 ed. SAS Inst., Cary, N.C.

Sherf, A.F. and A.A. MacNab. 1986. Vegetable diseases and their control. Wiley, New York.

Silue, D., N.I. Nashaat, and Y. Tirilly. 1996. Differential responses of Brassica oleracea and $B$. rapa accessions to seven isolates of Peronospora parasitica at the cotyledon stage. Plant Dis. 80:142-144.

Thomas, C.E. and L. Jourdain. 1989. Broccoli (Brassica oleracea) and downy mildew (Peronospora parasitica). Biol. and Cultural Tests for Control of Plant Dis. 4:11.

Thomas, C.E. and L. Jourdain. 1990. Evaluation of broccoli and cauliflower germplasm for resistance to race 2 of Peronospora parasitica. HortScience 25:1429-1431.

Wang, M. and M.W. Farnham. 1999. Ploidy of broccoli regenerated from microspore culture versus anther culture. Plant Breeding 118:249252.

Williams, P.H. 1986. Crucifer genetic resource book. Dept. Plant Pathology, Univ. of Wisconsin, Madison. 